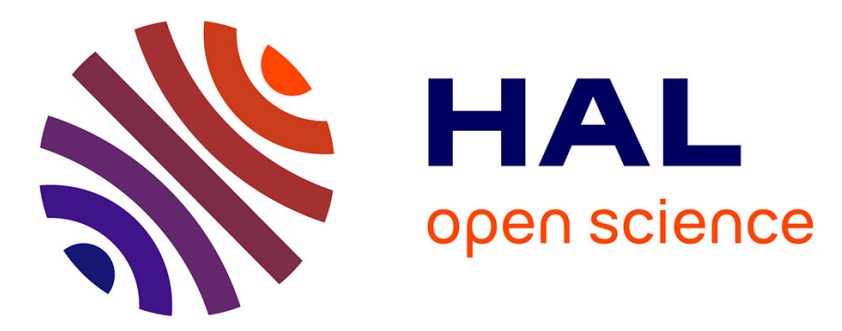

\title{
On a systematic approach for cracked rotating shaft study: breathing mechanism, dynamics and instability
}

\author{
Saber El Arem, Maha Ben Zid
}

\section{To cite this version:}

Saber El Arem, Maha Ben Zid. On a systematic approach for cracked rotating shaft study: breathing mechanism, dynamics and instability. Nonlinear Dynamics, 2017, 88 (3), pp.2123-2138. 10.1007/s11071-017-3367-7 . hal-02486672

\section{HAL Id: hal-02486672 \\ https://hal.science/hal-02486672}

Submitted on 21 Feb 2020

HAL is a multi-disciplinary open access archive for the deposit and dissemination of scientific research documents, whether they are published or not. The documents may come from teaching and research institutions in France or abroad, or from public or private research centers.
L'archive ouverte pluridisciplinaire HAL, est destinée au dépôt et à la diffusion de documents scientifiques de niveau recherche, publiés ou non, émanant des établissements d'enseignement et de recherche français ou étrangers, des laboratoires publics ou privés. 


\title{
On a systematic approach for cracked rotating shaft study breathing mechanism, dynamics and instability
}

\author{
Saber EL AREM · Maha BEN ZID
}

Received: date / Accepted: date

\begin{abstract}
We present a systematic approach to deal with the modeling and analysis of the cracked rotating shafts behaviour. We begin by revisiting the problem of modelling the breathing mechanism of the crack. Here we consider an original approach based on the form we give to the energy of the system and then identify the mechanism parameters using 3D computations with unilateral contact conditions on the crack lips. A dimensionless flexibility is identified which makes the application of the approach to similar problems straightforward. The additional flexibility due to the crack is then introduced in a simple and comprehensive dynamical system (2 DOF) to characterize the crack effects on the dynamical response of a rotating shaft. Many results could help in early crack detection.
\end{abstract}

Keywords cracked shafts $\cdot$ breathing crack $\cdot$ rotordynamics $\cdot$ instability

\section{Introduction}

Crack initiation in structural or equipment components may be caused by different mechanisms such as material defects or surface imperfections. Once initiated, a crack propagation is influenced by the loading, the material properties and the environment. In power plants, rotating turbines operate in highly stressed conditions and harsh environment prone to cracks initiation and propagation. In order to avoid catastrophic failures within parts of these important and very expensive structures, accurate vibration analyses have to be performed for early crack detection.

In dealing with cracked shafts mechanics, we mainly distinguich two aspects:

S. El Arem

Arts et Métiers ParisTech

Laboratoire LAMPA, CER Angers, France

E-mail: saber.elarem@ensam.eu

M. Ben Zid

Institut Universitaire de Technologie de Saint-Malo, France

Département Réseaux et Télécommunications 


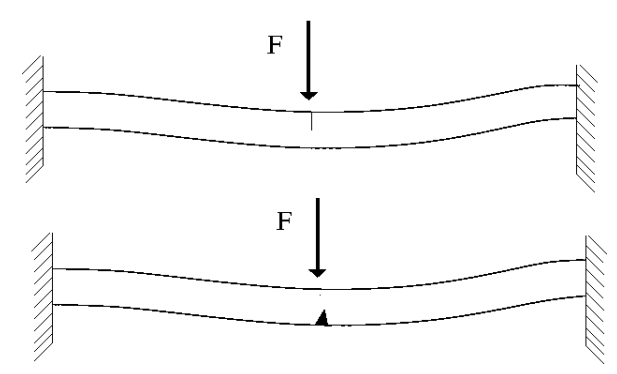

Fig. 1 Aspects of the crack breathing mechanism

1. The first one is to model the effects of the crack presence on the static behaviour of the shaft. When the shaft rotates, the crack opens and closes (breathes) and the resulting additional flexibility should be identified for all angular positions. The modeling and identification of this breathing mechanism are crucial since it measurably affects the dynamics of the system.

2. The second is the examination of the vibrational response of the system to clearly identify the effects of the crack presence and suggest parameters that could help in developping an efficient methodology for early crack detection.

The crack breathing mechanism: When the shaft rotates, the crack opens and closes depending on the local stress acting on the cracked transverse section. This behaviour is called breathing mechanism of the crack. By means of 3D non-linear models, Bachschmid et al [4] accurately investigated this mechanism and considered different crack geometries and loadings.

To develop a reliable model capable of predicting the vibrational response of a cracked shaft, this behaviour has to be identified accurately. There have been different approches to consider the local flexibility due to a crack affecting a shaft transverse section. Clearly, we could distinguish three of them.

1. The first and oldest one is based on fracture mechanics theory. It has been more than four decades since the Strain Energy Release Rate (SERR) theory has met with rotordynamics in the power industry when the detection of fatigue cracking became a necessity in power plants. Initiated in the 1970's by Dimarogonas and his co-workers, it is the most used approach in dealing with the bending of cracked shafts (Dimarogonas [9], Wauer [30]). A good review on this approach has been presented by Papadopoulos [27]. For an open crack, this method allows the calculation of the additional flexibility due to the crack presence (Dimarogonas and Papadopoulos [10]). Also, the nodal representation of the cracked transverse section takes into account the local aspect of cracking. However, errors arise when the breathing mechanism of the crack is considered and modification to the original method has been presented in (Darpe et al [8]). When the crack depth exeeds the transverse section radius, the SERR approach could not be used as discussed in Papadopoulos [26].

2. The second one is an original method for deriving a lumped model (an element of zero length) for a cracked beam transverse section that was developed by An- 
drieux and Varé [2] at Electricité De France (EDF). Based on 3D computations, the procedure incorporates more realistic behaviour of the crack than previous models and allows partial openning/closing. The authors established properties of the potential energy of the problem to reduce the amount of computations required for the model parameters identification. The experimental validation of the approach was presented by Stoisser and Audebert [29]. In using this approach in dynamics analysis of cracked shafts at Solid Mechanics Laboratory (LMS) of Ecole Polytechnique, El Arem [11] faced the problem of inverting the flexibility matrix when the crack is completely closed (additional flexibility is zero). To overcome this difficulty, El Arem and Maitournam [14] presented an elegant method to construct a cracked beam finite element to examine vibrational behaviour of rotating shafts. By distributing the additional energy due to the crack on the entire length of the element, a considerable gain in computing efforts was reached. This EDF-LMS approach is based on an energy formulation of the problem of identification of the breathing mechanism by means of 3D computations. The 3D modelling of the crack allows to get a fine and precise representation of its nonlinear behaviour. Also, with the simplicity of the quadratic form proposed for the system energy, we have significantly reduced the computational cost of the identification process. In fact, the breathing mechanism is completely described by the evolution with loading of one single (flexibility) function. With the EDF-LMS modelling procedures, it is possible to opt for a nodal representation of the cracked section (Andrieux and Varé [2], El Arem et al [16], El Arem [12], El Arem and Nguyen [15]) or to consider a beam element with a crack at midspan (El Arem and Maitournam [13], El Arem and Maitournam [14]). With the second choice, the energy due to the crack is distributed on the entire beam element.

3. The third approach has been developped by Bachschmid and his co-workers and called FLEX model which is described in detail in Bachschmid et al [3]. In the book of Bachschmid et al [5], comparisons of the FLEX model to the SERR and 3D have been carried out and showed that FLEX model approach seems to be more accurate. The method consists in identifying a beam model based on 3D computations. With the 3D computations, the breathing mechanism and the second moments of area are defined for the different angular positions of the crack. The stiffness matrix of an equivalent cracked beam element of suitable length $l_{c}$ is then calculated, assuming a Timoshenko beam with constant section and second moments of area along $l_{c}$. This method is similar to the model developped at Electricité de France (EDF) in many aspects. In fact, in both approches authors identified a beam model based on 3D computation of the additional flexibility due to the crack. The main difference is that Andrieux and Varé [2] presented a nodal modelling of the cracked section. Bachschmid defined an equivalent cracked beam element with length $l_{c}$ depending on the crack depth. We think that this approach is similar to the one adopted by El Arem and Maitournam [14] where the finite element length is fixed for all the angular positions of the crack as per the saintvenant theorem. The starting point for both approaches is the 3D modelling of the shaft element to identify the breathing mechanism. 
The approach presented in this article inherits from the EDF-LMS modeling procedures since it is based on the form chosen for the energy of the system allowing to distinguish the additional contribution due to the crack presence from that of the uncracked parts. Also, we concentrate the additional flexibility related to the crack in one single parameter (function) that depends on the system response (nonlinear). But, in this work, we identify a cracked beam element which is different from the nodal representation adopted in Andrieux and Varé [2]. Second, we present a dimensionless flexibility due to the crack wich is dependent only on the crack properties (geometry, relative depth) that can be used straightforward and without any additional 3D computations in similar problems. And, to make this approach generic and easy to adopt by engineers and scientists, we give an explicit polynomial function that approximates the additional flexibility due to the cracked transverse section. Thus, the procedure of identification could be skipped by those who will have adopted the approach described here, and this represents an important saving of time for scientists and powerplant operators. Our main objective in this article is to present a methodological approach in dealing with cracked shafts. We begin by revisiting the problem of determination of the breathing mechanism of the crack and explain all the steps up to the stability analysis. By analyzing the vibratory response of the cracked structure, we try to determine the parameters that can reveal the presence of a propagating crack.

\section{Breathing mechanism modeling and identification}

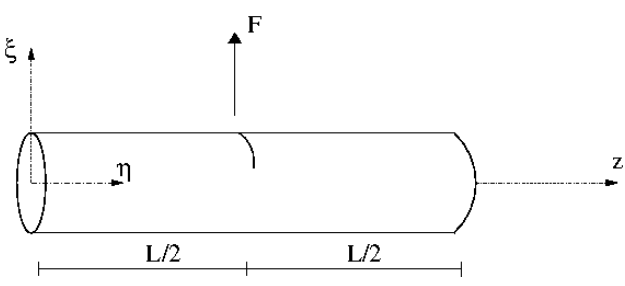

(a) Three-dimensional model

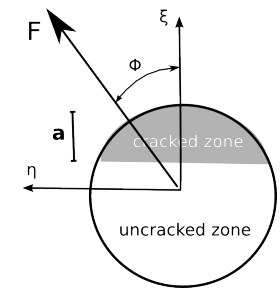

(b) Cracked transverse section

Fig. 2 The current $3 D$ model

The three-dimensional FE model considered is that of a cylinder of axis $(o z)$, radius $R$, length $L$, containing, at midspan, a cracked transverse section, cf. Figure 2. The structural element, clamped at its both ends $(z=0$ and $z=L)$, is subjected at $z=\frac{L}{2}$ to a force $\mathbf{F}=\left(F_{\xi}, F_{\eta}\right)$. The following assumptions are considered for the identification of the breathing mechanism of the crack:

1. small displacements and small deformations,

2. linear elastic and isotropic material,

3. transverse cracks of any shape and in any number, 
4. unilateral contact without friction between the crack lips,

5. the crack is completely closed in the unstressed configuration.

Let $W^{*}$ be the total elastic (complementary) energy of the system. According to Andrieux and Varé [2] and El Arem [11], $W^{*}$ can be put in the form:

$$
W^{*}(\mathbf{F})=W_{s}^{*}(\mathbf{F})+w_{c}^{*}(\mathbf{F})
$$

where $W_{s}^{*}$ denotes the total elastic energy of the uncracked structure under the loading $\mathbf{F}$, and $w_{c}^{*}(\mathbf{F})$ the additional elastic energy due to the presence of the crack. Some properties of the problem energy will make easier the identification of the flexibility due to the crack presence (Andrieux and Varé [2], El Arem [11]).

\section{Property 1:}

$w_{c}^{*}$ is strictly convex and positively homogeneous of degree $\mathbf{2}$ :

$$
\forall \lambda \geq 0, w_{c}^{*}(\lambda \mathbf{F})=\lambda^{2} w_{c}^{*}(\mathbf{F})
$$

Property 1 comes from the fact that the contact surface between the crack lips (and therefore the additional flexibility) does not depend on $\|F\|$ but only on the direction of $\mathbf{F}$. It should be noticed that an essential hypothesis for obtaining propertie 1 is that the gap between the lips of the crack is zero in the unstressed configuration which distinguishes the crack from a notch.

A raisonable choice for $w_{c}^{*}$ is to consider a quadratic form of $\mathbf{F}$. We write:

$$
w_{c}^{*}(\mathbf{F})=\frac{1}{2} s_{c}(\mathbf{F})|| \mathbf{F} \|^{2}
$$

$s_{c}(\mathbf{F})$ represents the additional flexibility of the structure due to the crack presence to be identified by means of $3 \mathrm{D}$ computations.

With Property 1, the problem of identification of the function $w_{c}^{*}$ on $\mathbb{R}^{2}$ is reduced to the identification of the flexibility function $s_{c}(\Phi)$ on the interval $[0,2 \pi]$ by considering :

$$
\mathbf{F}=\left(F_{\xi}, F_{\eta}\right)=(\cos (\Phi), \sin (\Phi)),\|F\|=1 \text { and } \Phi=\operatorname{atan}\left(\frac{F_{\eta}}{F_{\xi}}\right)
$$

The total elastic energy of the system could be written:

$$
W^{*}(\mathbf{F})=\frac{1}{2} s(\Phi)\|\mathbf{F}\|^{2}=\frac{1}{2}\left\{s_{0}+s_{c}(\Phi)\right\}\|\mathbf{F}\|^{2}
$$

where $s_{0}$ is a constant representing the bending flexibility of the uncracked shaft. Instead of identifying the function $s_{c}(\Phi)$, it would be more advantageous to identify a parameter that lets appear the intrinsic properties of the crack. Thus, a dimensionless coefficient that only depends on the crack parameters (geometry, depth, etc...) would be more useful to identify. This aims to make our approach generic and easily exploitable in similar configurations. Dimarogonas and Papadopoulos [10] presented 

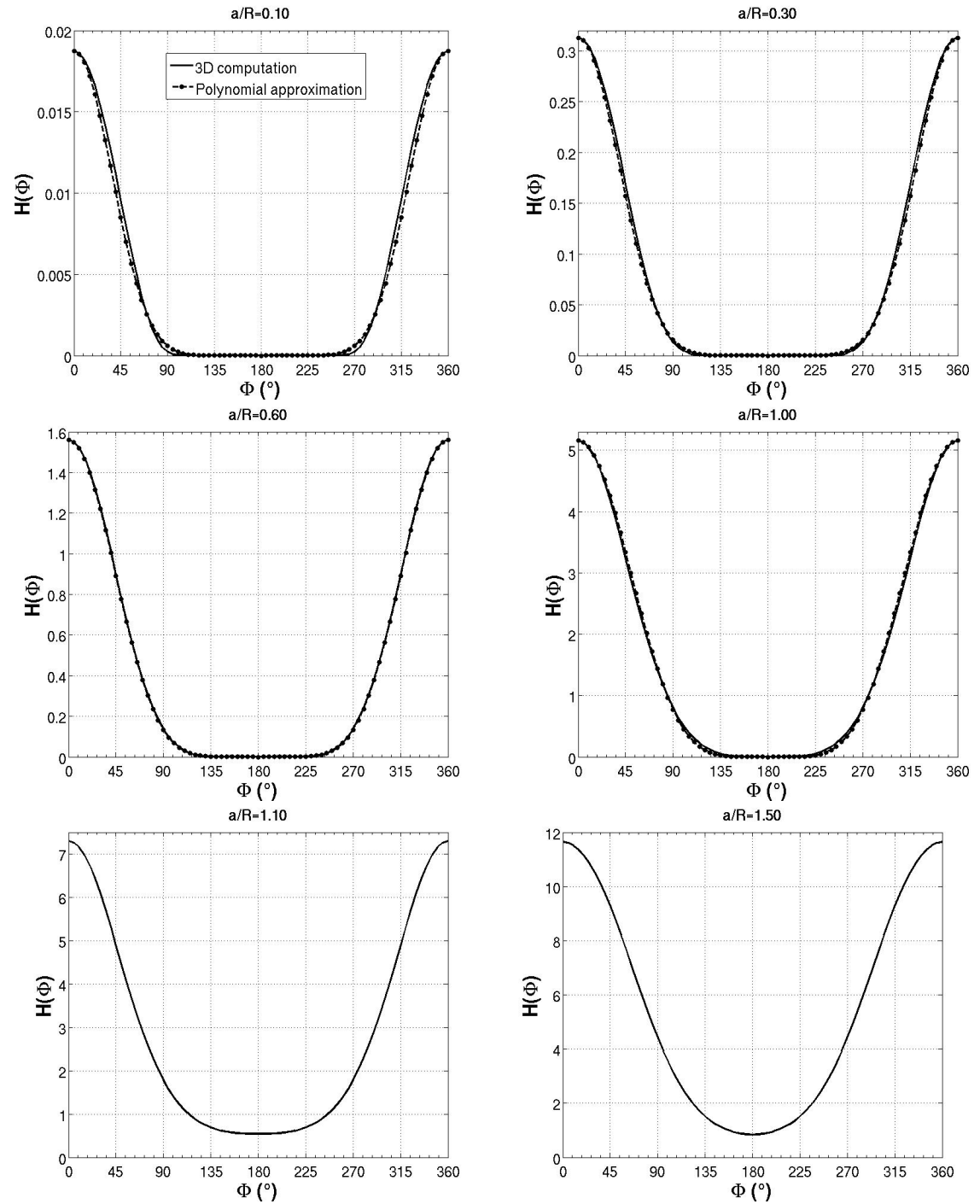

Fig. 3 Dimensionless flexibility as a function of loading angle for different crack depth

a dimensionless flexibility computed numerically and based on the fracture mechanics approach. Also Gasch [18] considered a dimensionless flexibility of the crack that was obtained experimentally. For these reasons, we write:

$$
W^{*}(\mathbf{F})=\frac{1}{2} s(\Phi)\|\mathbf{F}\|^{2}=\frac{1}{2}\left\{s_{0}+\frac{L^{2}}{48 \pi E R^{3}} H(\Phi)\right\}\|\mathbf{F}\|^{2}
$$

Given a crack geometry and a loading direction $\Phi$, the dimensionless flexibility $H$ represents a measure of the open (closed) parts of the crack. $E$ is the Young's modulus of the material. 
2.1 Identification procedure of the dimensionless flexibility $H$ :

Three-dimensional computations have been carried out to determine $H$ for the case presented in Figure 2. The structure contains a crack with a rectilinear tip at midspan. The clamped shaft element is subjected to $\mathbf{F}$ and the angle $\Phi$ is varied in $\left[0^{\circ}, 360^{\circ}\right]$ at a rate of a loading case every $5^{\circ}$ and, thus, a total of 72 loading cases where carried out. The identification of $H$ also requires the realization of similar computations on the uncracked structure. Knowing the forces $(\mathbf{F})$ and the displacements $(\mathbf{u})$ at the cracked section, the formula of Clapeyron makes it easy to evaluate $W_{s}^{*}$ and $W^{*}$ of the uncracked and the cracked structures, respectively (El Arem [12], El Arem and Maitournam [14]). $w_{c}^{*}$ is obtained by:

$$
w_{c}^{*}(\Phi)=W^{*}(\Phi)-W_{s}^{*}(\Phi), \forall \Phi \in[0,360]
$$

then $H$ is computed using:

$$
H(\Phi)=w_{c}^{*}(\Phi) \frac{48 \pi E R^{3}}{L^{2}}, \forall \Phi \in[0,360]
$$

Figure 3 shows that for cracks with a rectilinear tip and a relative depth $\frac{a}{R}<1.0$, the crack is fully open when $\Phi=0^{\circ}$ (or $360^{\circ}$ ) and closes completely on an interval arround $\Phi=180^{\circ}$. In fact, despite the rotation, shallow cracks may remain totally into the compressed half-section before going into the taut zone. This interval reduces to $\Phi=180^{\circ}$ when $\frac{a}{R}=1$. Between these loading cases, the crack is partially open. The evolution from totally open to totally closed crack is smooth and regular.

When $\frac{a}{R}>1.0$, the crack can not be fully closed since it can not be completely contained in the compressed half-section for any $\Phi \in\left[0,360^{\circ}\right]$. In this case, $H(\Phi)>0$ and its lowest value is reached at $\Phi=180^{\circ}$ as shown in Figure 3 for $\frac{a}{R}=1.10$ and $\frac{a}{R}=1.50$.

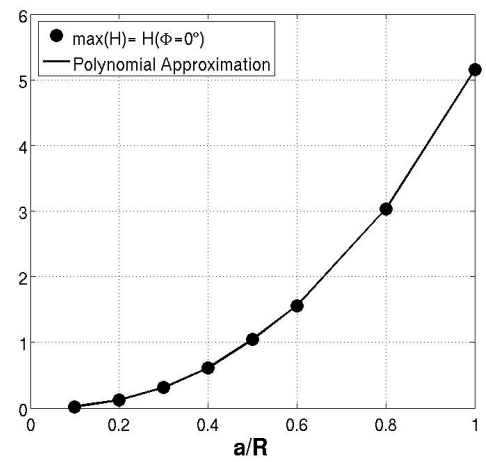

Fig. 4 Maximum of dimensionless flexibility as a function of crack depth

In Figure 4 we present the maximum of additional flexibility $(\max (H))$ as a function of the relative depth of the $\operatorname{crack}\left(\frac{a}{R}\right)$. By adopting a polynomial fitting we wrote: 


$$
\max (H)\left(\frac{a}{R}\right)=P_{a}\left(\frac{a}{R}\right)=\sum_{i=0}^{7} c_{i}\left(\frac{a}{R}\right)^{i}
$$

Also, we have found that a good fitting of the values of $H$ would be:

$$
H(\Phi)=\max (H)\left\|\sin \left(\frac{\Phi+\pi}{2}\right)\right\|^{Q_{a}\left(\frac{a}{R}\right)}=P_{a}\left(\frac{a}{R}\right)\left\|\sin \left(\frac{\Phi+\pi}{2}\right)\right\|^{Q_{a}\left(\frac{a}{R}\right)}
$$

with $Q$ a polynomial function of $\frac{a}{R}$ given by:

$$
Q_{a}\left(\frac{a}{R}\right)=\sum_{i=0}^{7} q_{i}\left(\frac{a}{R}\right)^{i}
$$

In Figure 3 we can see that the polynomial approximation of $H$ using (9) produces an excellent fitting for cracks with rectilinear tip and width $\frac{a}{R} \leq 1.0$. We can easily extend the approach for deeper cracks by considering a formula similar to (9) and adding a constant term (which is $\min (H)=H(\Phi=\pi)$ ) that increases with the crack depth. In this first article devoted to this new systematic approach, we want to focus on shallower cracks since we aim to characterize cracks effects before they become of critical depth.

Table 1 Coefficients of the polynomial functions $P_{a}$ and $Q_{a}$

\begin{tabular}{lllllllll}
\hline & $\mathrm{i}=0$ & $\mathrm{i}=1$ & $\mathrm{i}=2$ & $\mathrm{i}=3$ & $\mathrm{i}=4$ & $\mathrm{i}=5$ & $\mathrm{i}=6$ & $\mathrm{i}=7$ \\
\hline$c_{i}$ & 0.84 & -20.9 & 197.30 & -902.48 & 2309.70 & -3258.93 & 2371.48 & -691.79 \\
$q_{i}$ & 8.87 & 48.35 & -626.78 & 3386.94 & -9610.24 & 14657.99 & -11307.04 & 3447.42 \\
\hline
\end{tabular}

\subsection{Constitutive equations}

As mentioned earlier, our objective is to build a beam model based on realistic 3D model to be extensively used in the nonlinear dynamics of cracked rotating shafts. The nonlinear constitutive equations at the cracked section (midspan) are obtained by differentiating $W^{*}$ with respect to $\mathbf{F}$. we write:

$\mathbf{u}=\left(\begin{array}{c}u_{\xi} \\ u_{\eta}\end{array}\right)=\mathbf{S}(\Phi)\left(\begin{array}{c}F_{\xi} \\ F_{\eta}\end{array}\right)=\left\{\mathbf{S}_{\mathbf{0}}+\mathbf{S}_{\mathbf{c}}(\Phi)\right\}\left(\begin{array}{c}F_{\xi} \\ F_{\eta}\end{array}\right)=\left(\begin{array}{cc}s_{0}+s_{c}(\Phi) & -\frac{1}{2} s_{c}^{\prime}(\Phi) \\ \frac{1}{2} s_{c}^{\prime}(\Phi) & s_{0}+s_{c}(\Phi)\end{array}\right)\left(\begin{array}{c}F_{\xi} \\ F_{\eta}\end{array}\right)$

where $\mathbf{S}_{\mathbf{0}}$ and $\mathbf{S}$ are, respectively, the flexibility matrices of the uncracked and cracked shaft.

$$
s_{c}^{\prime}(\Phi)=\frac{d s_{c}(\Phi)}{d \Phi}=\frac{L^{2}}{48 \pi E R^{3}} \frac{d H(\Phi)}{d \Phi}=\frac{L^{2}}{48 \pi E R^{3}} H^{\prime}(\Phi)
$$


Finally, at the cracked section we have:

$$
\left(\begin{array}{l}
u_{\xi} \\
u_{\eta}
\end{array}\right)=\left\{\left(\begin{array}{cc}
s_{0} & 0 \\
0 & s_{0}
\end{array}\right)+\frac{L^{2}}{48 \pi E R^{3}}\left(\begin{array}{cc}
H(\Phi) & -\frac{1}{2} H^{\prime}(\Phi) \\
\frac{1}{2} H^{\prime}(\Phi) & H(\Phi)
\end{array}\right)\right\}\left(\begin{array}{l}
F_{\xi} \\
F_{\eta}
\end{array}\right)
$$

The extradiagonal terms show that the crack introduces coupling between the transverse directions of the shaft element. In numerical codes for structural analysis, we usually prefer a relation of the form:

$$
\left(\begin{array}{c}
F_{\xi} \\
F_{\eta}
\end{array}\right)=\{\mathbf{S}(\Phi)\}^{-1}\left(\begin{array}{c}
u_{\xi} \\
u_{\eta}
\end{array}\right)=\mathbf{K}(\mathbf{u})\left(\begin{array}{c}
u_{\xi} \\
u_{\eta}
\end{array}\right)=\left\{\mathbf{K}_{\mathbf{0}}-\mathbf{K}_{\mathbf{c}}(\mathbf{u})\right\}\left(\begin{array}{c}
u_{\xi} \\
u_{\eta}
\end{array}\right)
$$

where $\mathbf{K}_{\mathbf{0}}$ and $\mathbf{K}$ are, respectively, the stiffness matrices of the uncracked and cracked shaft, with

$$
\mathbf{K}_{\mathbf{0}}=\left(\begin{array}{cc}
k_{0} & 0 \\
0 & k_{0}
\end{array}\right) \text { and } k_{0}=\frac{1}{s_{0}}
$$

$\mathbf{K}_{\mathbf{c}}(\mathbf{u})$ represents the stiffness loss due to the crack and depending on the problem unknowns $\mathbf{u}$ which makes the problem nonlinear. Actually, for a given loading direction $\Phi$, the problem is linear with different stiffness for each angle $\Phi$. This fact is well presented by Figure 5 where we can see that the loading angle $\Phi$ is equal to the response angle $\psi$ defined by:

$$
\psi=\operatorname{atan}\left(\frac{u_{\eta}}{u_{\xi}}\right)
$$

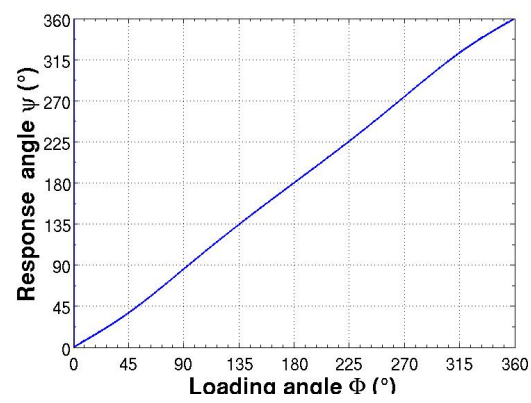

Fig. 5 System bilinearity $\Phi=\psi$.

By writting $\mathbf{K}_{\mathbf{c}}(\mathbf{u})$ in the form:

$$
\mathbf{K}_{\mathbf{c}}=\frac{48 \pi E R^{3}}{L^{2}}\left(\begin{array}{l}
k_{\xi \xi}(\psi) k_{\xi \eta}(\psi) \\
k_{\eta \xi}(\psi) k_{\eta \eta}(\psi)
\end{array}\right)
$$


we obtain:

$$
\begin{aligned}
& k_{\xi \xi}(\psi)=k_{\eta \eta}(\psi)=\frac{R}{L}-\frac{4 R(L+R H(\Phi))}{\left(4 R^{2} H(\Phi)^{2}+8 L R H(\Phi)+R^{2} H^{\prime}(\Phi)^{2}+4 L^{2}\right)} \\
& k_{\xi \eta}(\psi)=-k_{\eta \xi}(\psi)=-\frac{2 R^{2} H^{\prime}(\Phi)}{\left(4 R^{2} H(\Phi)^{2}+8 L R H(\Phi)+R^{2} H^{\prime}(\Phi)^{2}+4 L^{2}\right)}
\end{aligned}
$$

We can notice that

$$
k_{\xi \eta}(\psi)=-\frac{1}{2} \frac{d k_{\xi \xi}}{d \psi}=-\frac{1}{2} k_{\xi \xi}^{\prime}
$$

Also, when $H(\Phi)=H^{\prime}(\Phi)=0$ (no crack), we have:

$$
k_{\xi \xi}(\psi)=k_{\xi \eta}(\psi)=0
$$

Thus, $\mathbf{K}_{\mathbf{c}}$ can be put in the form:

$$
\mathbf{K}_{\mathbf{c}}=\frac{48 \pi E R^{3}}{L^{2}}\left(\begin{array}{cc}
k_{\xi \xi}(\psi) & -\frac{1}{2} k_{\xi \xi}^{\prime}(\psi) \\
\frac{1}{2} k_{\xi \xi}^{\prime}(\psi) & k_{\xi \xi}(\psi)
\end{array}\right)
$$

Like $\mathbf{S}_{\mathbf{c}}, \mathbf{K}_{\mathbf{c}}$ is a skew-symmetric matrix. Also, we can easily notice that the stiffness matrix of cracked transverse section is completely identified by one single function $k_{\xi \xi}$ and its derivative.

Finaly, at the cracked transverse section, we have the relation:

$$
\left(\begin{array}{c}
F_{\xi} \\
F_{\eta}
\end{array}\right)=\left\{\left(\begin{array}{cc}
k_{0} & 0 \\
0 & k_{0}
\end{array}\right)-\frac{48 \pi E R^{3}}{L^{2}}\left(\begin{array}{cc}
k_{\xi \xi}(\psi) & -\frac{1}{2} k_{\xi \xi}^{\prime}(\psi) \\
\frac{1}{2} k_{\xi \xi}^{\prime}(\psi) & k_{\xi \xi}(\psi)
\end{array}\right)\right\}\left(\begin{array}{l}
u_{\xi} \\
u_{\eta}
\end{array}\right)
$$

with $k_{0}=\frac{192 E I}{L^{3}}=\frac{48 \pi E R^{4}}{L^{3}}$ the bending stiffness of a biclamped uniform beam. $I=\frac{\pi R^{4}}{4}$ is the quadratic moment of inertia.

\subsection{Validation of the approach}

In this section we are going to compare the $3 \mathrm{D}$ computations results to the ones obtained using a beam model. Both models represent a structure clamped at its both ends and containing a cracked transverse section at midspan. In the beam model we use the flexibility $(H)$ identified from $3 \mathrm{D}$ computations as described in the previous section. Once identified, $H$ could be used in any configuration where the crack has the same shape and relative depth. In our identification procedure, we have considered 


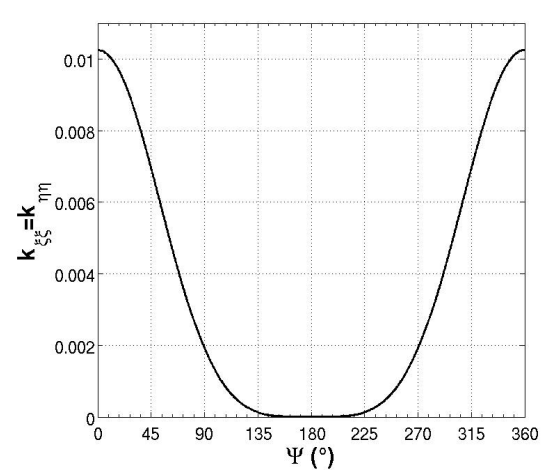

(a)

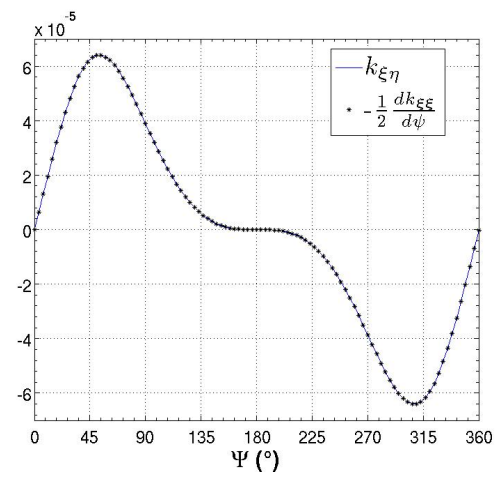

(b)

Fig. 6 Stiffness loss due to the crack for $\frac{a}{R}=1$ : (a) $k_{\xi \xi}$. (b) $k_{\xi \eta}$
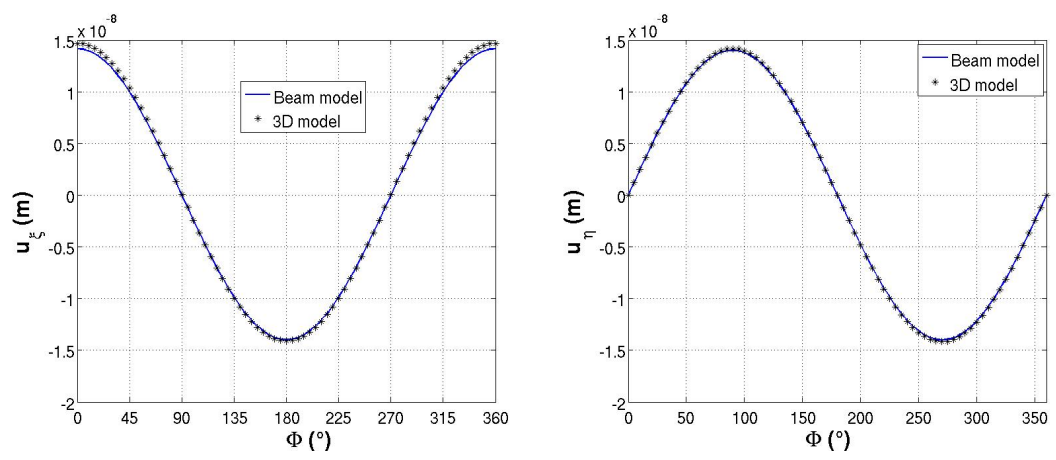

Fig. 7 Validation of the procedure. $\frac{a}{R}=1.0$

a cylinder of radius $R=0.5 \mathrm{~m}$, length $L=10 \mathrm{~m}$ containing, at midspan, a crack of rectilinear tip with relative depth $\frac{a}{R}=1.0$. For the beam model to be validated, we consider a different structure with $R=0.25 \mathrm{~m}$, length $L=12 \mathrm{~m}$ and we suppose that a crack with the same geometry as the one used in the identification procedure $\left(\frac{a}{R}=\right.$ 1.0 ) is located at the midspan. Knowing the loading force $\mathbf{F}$, we use equation (7) with the dimensionless flexibility $H$ to compute the displacement $\mathbf{u}$ at the cracked section. These displacements are then compared to the results obtained with a complete 3D model of a cylinder of radius $R=0.25 \mathrm{~m}$, length $L=12 \mathrm{~m}$ containing, at midspan, a crack with $\left(\frac{a}{R}=1.0\right)$.

Figure 7 shows an exellent agreement between the 3D results and the beam model. Although the 3D computations cosume less than 10 minutes of CPU time, having a reliable and robust beam model is always preferable especially for the examination of the nonlinear dynamics of the system as will be discussed in the next section. 


\section{Nonlinear dynamics of a rotating shaft with a breathing crack}

This section is devoted to the examination of the vibrational response of a De Laval rotor (Figure 8) with the breathing mechanism identified in the first part of this article. The mass of the shaft is assumed to be negligeable compared to the disc mass. At the begining of cracked shafts dynamics analysis, many authors (Gasch [18], Mayes and Davies [22], Grabowski [20]) considered the breathing mechanism of the crack as depending on the angular position $(\Omega t)$ only. This simplification comes from the implicit hypothesis of an unchanged location of the taut zone of the transverse section upon rotation of the shaft. In fact, for horizontal axis shafts, the weight deflection is dominating and it governs the opening and closing of the crack since the vibration amplitudes are small compared to the static deflection. As part of this widespread assumption, the lower half of the shaft transverse section is taut while the upper half is compressed as presented with position $I$ in Figure 9 . And the cracked portion passes in one zone then the other when the shaft rotates and thus opens and closes periodically. This means that :

$$
\overrightarrow{O G} \approx\|\overrightarrow{O G}\| \overrightarrow{O x} \quad \forall t
$$

This hypothesis leads to a linear system of equations with periodic coefficients. However, to build a systematic approach, we need to remove all the non essential assumptions. We want our methodology to be applicable in the most general configurations. In fact, the new generation of turbines are light weight and often operated at very high frequencies (many times the first critical speed) resulting in hight levels of vibrations. Also in vertical axis machines, the machine self-weight is not the dominant loading. In such configurations, hypothesis of weight dominant situation could not be accepted and we need a robust model capable of handling all possible situations like transient conditions of starting up, coasting down or passing throught resonnance rotating frequencies. In these situations, the vibration levels effects could be the same order of magnitude than the self-weight deflection.

In the most general case, when considering the modeling of breathing mechanism of the crack in a rotating shaft, we need at each time instant to be able of answering two questions:

1. First, which part of the transverse section is under tension?

2. Second, where is the cracked part of the transverse section with respect to that taut zone?

To answer the first question, we need to know the position of the transverse section center $G$. Figure 9 shows the evolution of the taut zone (outer half-section) with $G$ position. The line of separation with the compressed zone is perpendicular to OG as per the elasticity theory.

The position of the crack is given by the rotation angle $\Omega t$. Knowing $(U, V)$ of $G$ and $\Omega t$, we can define in a unique way the stiffness of the structure (Figure 8). In fact,

$$
\psi=\operatorname{atan}\left(\frac{u_{\eta}}{u_{\xi}}\right)=\gamma-\Omega t=\operatorname{atan}\left(\frac{V}{U}\right)-\Omega t
$$

In the rotating, shaft fixed frame $O \xi \eta$, we have established the relation: 


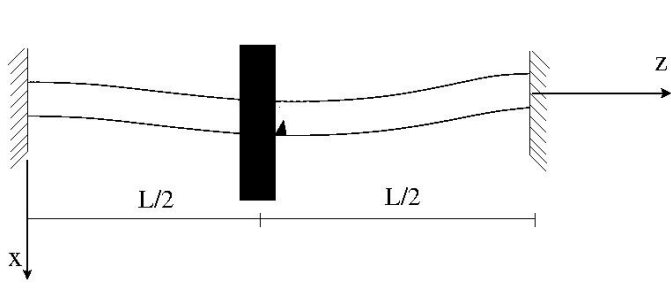

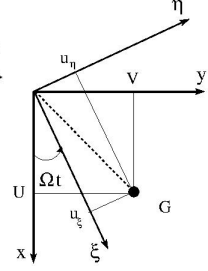

(b)

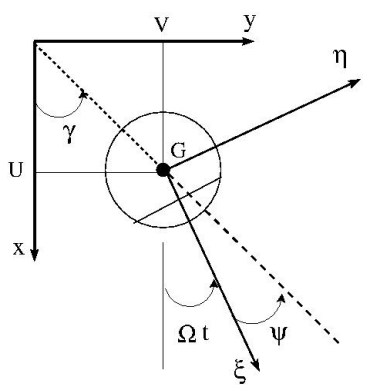

(c)

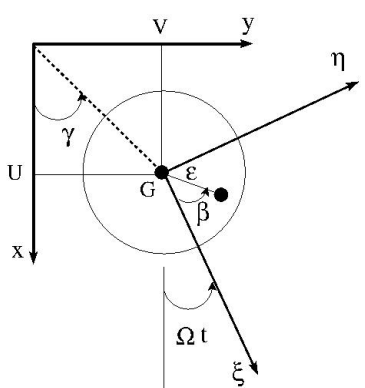

(d)

Fig. 8 Dynamical system. (a) DeLaval rotating shaft with crack at midspan. (b) -(d) System parameters.

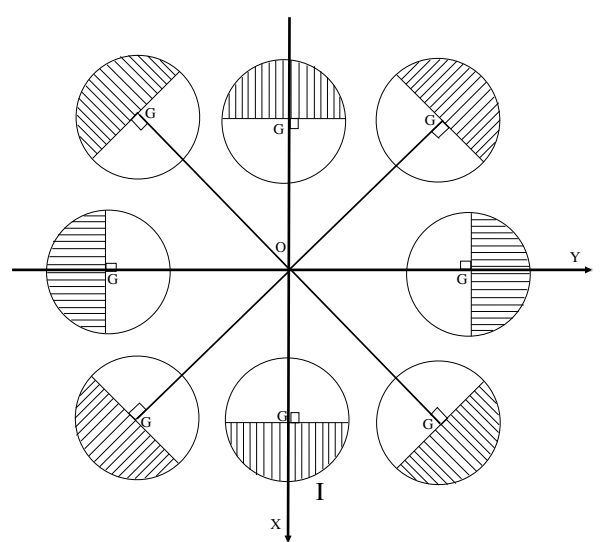

Fig. 9 Evolution of the Compressed and taut (hatched) parts of the transverse section with rotation

$$
\left(\begin{array}{c}
F_{\xi} \\
F_{\eta}
\end{array}\right)=\left\{\left(\begin{array}{cc}
k_{0} & 0 \\
0 & k_{0}
\end{array}\right)-\frac{48 \pi E R^{3}}{L^{2}}\left(\begin{array}{cc}
k_{\xi \xi}(\psi) & -\frac{1}{2} k_{\xi \xi}^{\prime}(\psi) \\
\frac{1}{2} k_{\xi \xi}^{\prime}(\psi) & k_{\xi \xi}(\psi)
\end{array}\right)\right\}\left(\begin{array}{l}
u_{\xi} \\
u_{\eta}
\end{array}\right)
$$

To shift to the inertial frame $O x y$, we write: 


$$
\mathbf{u}=\left(\begin{array}{l}
u_{\xi} \\
u_{\eta}
\end{array}\right)=\left(\begin{array}{cc}
\cos \Omega t & \sin \Omega t \\
-\sin \Omega t & \cos \Omega t
\end{array}\right)\left(\begin{array}{l}
U \\
V
\end{array}\right)=(T(\Omega t)) \mathbf{U}
$$

Analogously for the forces, we write:

$$
\mathbf{F}=\left(\begin{array}{c}
F_{\xi} \\
F_{\eta}
\end{array}\right)=\left(\begin{array}{cc}
\cos \Omega t & \sin \Omega t \\
-\sin \Omega t & \cos \Omega t
\end{array}\right)\left(\begin{array}{c}
P_{x} \\
P_{y}
\end{array}\right)=\mathbf{T}(\Omega t) \mathbf{P}
$$

Thus:

$$
\mathbf{P}=\mathbf{T}^{-\mathbf{1}}(\Omega t) \mathbf{F}
$$

Inserting these transformations of forces and deflections into equation (22), we obtain the stiffness matrix $\mathbb{K}(\mathbf{U})$ expressed in the inertial frame.

$$
\mathbb{K}(\mathbf{U})=\mathbf{T}^{-\mathbf{1}}(\Omega t) \mathbf{K}(\mathbf{u}) \mathbf{T}(\Omega t)
$$

The construction process of the stiffness matrix $\mathbf{K}(\mathbf{u})$ of the cracked shaft element lead to a skew-symmetric matrix:

$$
\mathbf{K}(\mathbf{u})=-\mathbf{K}^{t}(\mathbf{u})
$$

which is invariable by rotation. We have:

$$
\mathbb{K}(\mathbf{U})=\mathbf{K}(\mathbf{u})
$$

Now we can write the dynamical equilibrium equations of the DeLaval rotor of Figure 8 as given by the Principal of Virtual Power:

$$
\mathbf{M u ̈}+\mathbf{D} \dot{\mathbf{u}}+\mathbf{K}(\mathbf{u}) \dot{\mathbf{u}}=\mathbf{P}_{\mathbf{0}}+\mathbf{P}_{\mathbf{u}}
$$

or:

$$
\left(\begin{array}{l}
m \\
m
\end{array}\right)\left(\begin{array}{l}
\ddot{\mathbf{U}} \\
\ddot{\mathbf{V}}
\end{array}\right)+\left(\begin{array}{c}
D \\
D
\end{array}\right)\left(\begin{array}{l}
\dot{\mathbf{U}} \\
\dot{\mathbf{V}}
\end{array}\right)+\frac{48 \pi E R^{3}}{L^{2}}\left(\begin{array}{cc}
\frac{R}{L}-k_{\xi \xi}(\psi) & \frac{1}{2} k_{\xi \xi}^{\prime}(\psi) \\
-\frac{1}{2} k_{\xi \xi}^{\prime}(\psi) & \frac{R}{L}-k_{\xi \xi}(\psi)
\end{array}\right)\left(\begin{array}{l}
\mathbf{U} \\
\mathbf{V}
\end{array}\right)=\left(\begin{array}{c}
m g \\
0
\end{array}\right)+\varepsilon m_{u} \Omega^{2}\left(\begin{array}{c}
\cos (\beta+\Omega t) \\
\sin (\beta+\Omega t)
\end{array}\right)
$$

$\mathbf{P}_{\mathbf{0}}$ is the disc weight and $\mathbf{P}_{\mathbf{u}}$ is the umbalance forces due to the umbalance mass $m_{u}$ at $\varepsilon$ from $G$ (Figure 8).

$D=2 d m w_{0}$ is the viscous damping coefficient, $d$ the reduced (dimensionless) damping coefficient and $w_{0}=\sqrt{\frac{k_{0}}{m}}$ the natural frequency of the uncracked structure.

At $t=0$, the crack is totally open, cf. Figure 8 . We suppose that the stiffness matrix remains constant between two instants $t_{n}=n h$ and $t_{n+1}=(n+1) h$ where $h$ is the time step used for the numerical integration of the dynamical system. For the validity of this approximation, the time steps should be relatively small compared to the excitation period $\left(\frac{1}{\Omega}\right)$. In the low frequency domain, this difficulty could be easily 

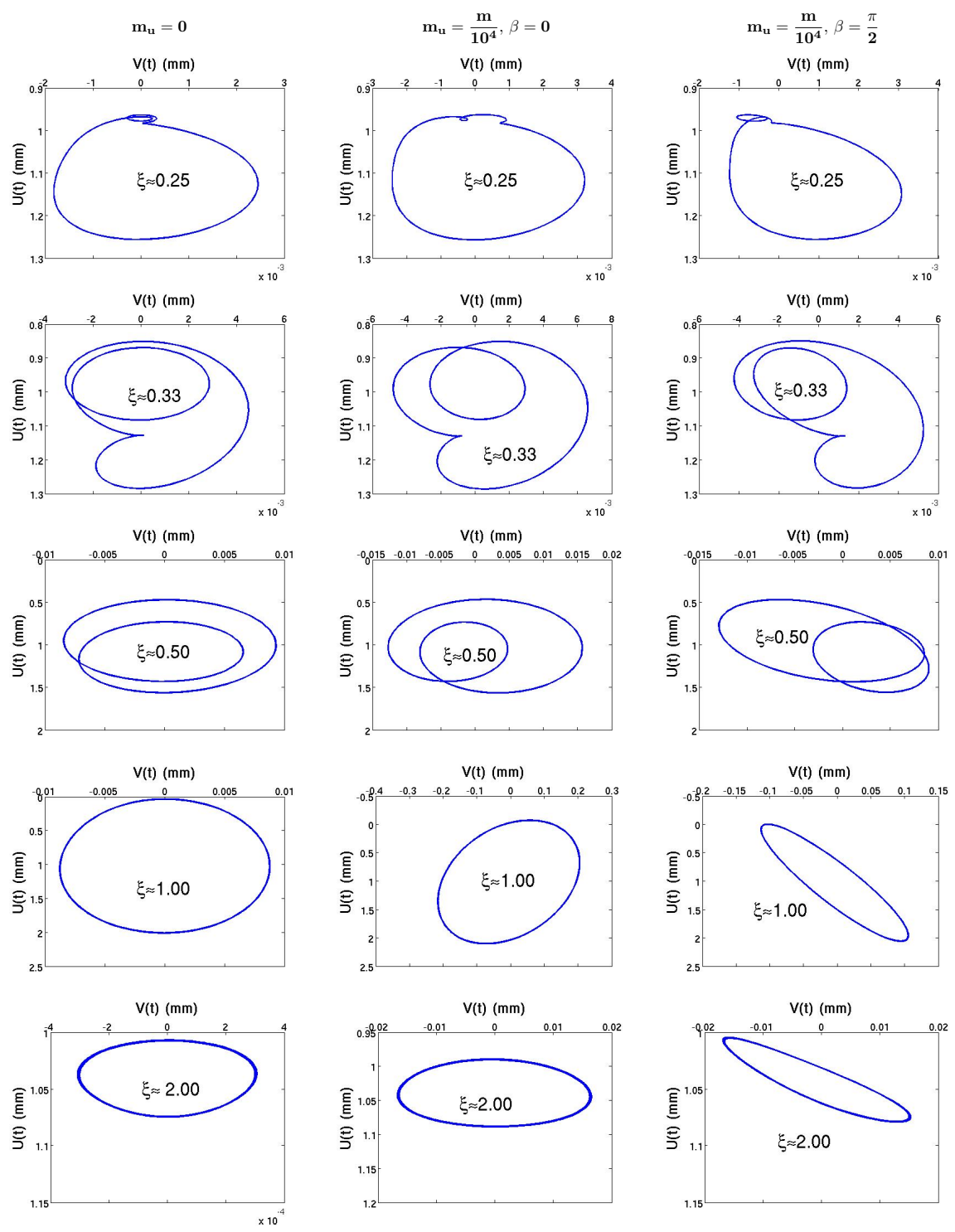

Fig. 10 Examples of the rotor orbits, $\xi=\frac{\Omega}{w_{0}}, \frac{a}{R}=1, d=0.03$

overcome. Thus, the stiffness matrix is updated at the end of each integration step. The Newmark method is adopted for the numerical integration of the obtained system 

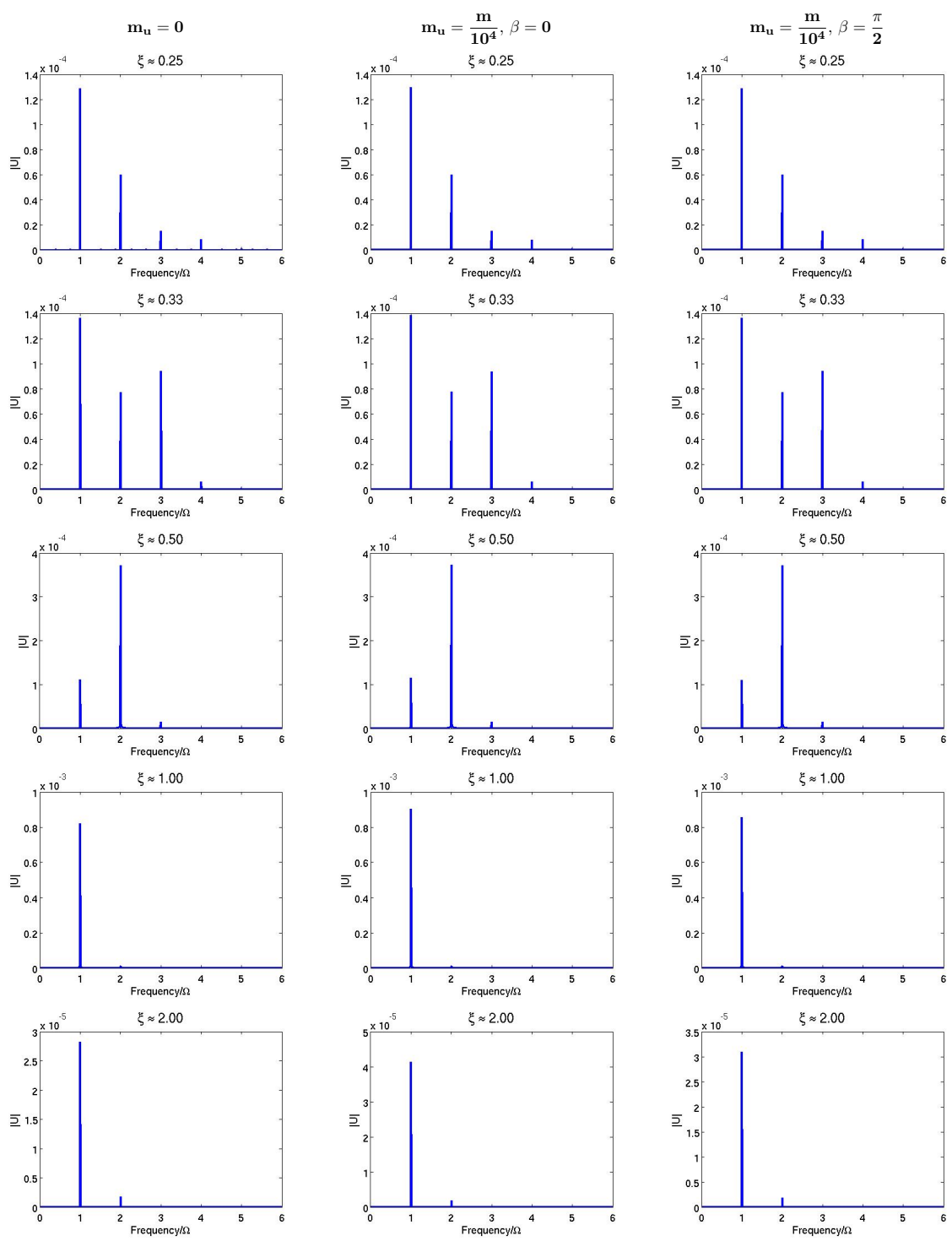

Fig. 11 Examples of amplitude spectra, $\xi=\frac{\Omega}{w_{0}}, \frac{a}{R}=1, d=0.03$

with:

$$
\gamma=\frac{1}{2} \text { and } \beta=\frac{1}{4}
$$


The shaft orbits presented in Figure 10 show that the superharmonic resonance phenomena presence when the rotating frequency passes through entire divisions of the critical speed $w_{0}$. Thus, at $\xi \approx \frac{1}{4}$ we have four loops per one revolution of the shaft, as this is the resonance of the four times per revolution excitation. The same phenomena could be observed with $\xi \approx \frac{1}{3}$ and $\xi \approx \frac{1}{2}$ where the orbit is composed of three and two loops, respectively.

When $\xi \approx \frac{\Omega}{n}$, the vibratory amplitude of $n^{\text {th }}$ harmonic reaches higher levels, cf. Figure 11. At starting up, coasting down the observation of this superharmonic resonances at subcritical rotor frequencies represents a good indication of crack presence as mentioned in by Gasch [19], El Arem and Maitournam [14], Patel and Darpe [28], AL-Shudeifat et al [1], El Arem and Nguyen [15]. The form of the rotor whirl orbit could be influenced by a little change in the system parameters like umbalance (Figure 10) or rotating frequency, etc. These changes could hide some loops of the orbit that are due to superharmonic resonnances. Consequently, we must rely more on information contained in the amplitude spectra because they are less affected by these small changes (Figure 11). In the normal operating conditions, the turbines are rotating at constant frequency, and here a crack growth could be depicted by monitoring the evolution of the static deflection and the levels of the first and second harmonics (todays vibration minus vibration a fortnight before), cf. Figure 12. These vibratory parameters are very reliable for early detecting the presence and propagation of cracks. Typical static and dynamical behaviour of cracked shafts has been presented by Bachschmid et al [6] who showed that measured vibrations could be successfully used to identify accurately crack position and depth.
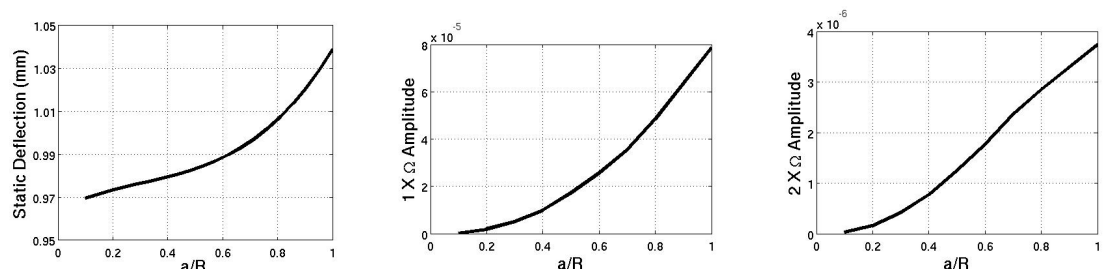

Fig. 12 Evolution of vibratory parameters with crack depth, $\xi=1.50, d=0.03$

\section{Stability analysis}

Parametric instability of a Jeffcott rotor with rotationally asymmetric inertia and transverse crack was carried out by Han and Chu [21]. The authors considered an open crack model and examined its influence on the stability of the system. Chen and Dai [7] explored the response of a cracked rotor system with viscoelastic supports. The authors observed bifurcation, $N T$-periodic, quasi-periodic and chaotic behaviours depending on the combinaisons of the system parameters.

In this work, the stability of the cracked shaft of Figure 8 containing a straight tip crack at mid-span is analyzed using the Floquet method (Floquet [17], Nayfeh and 

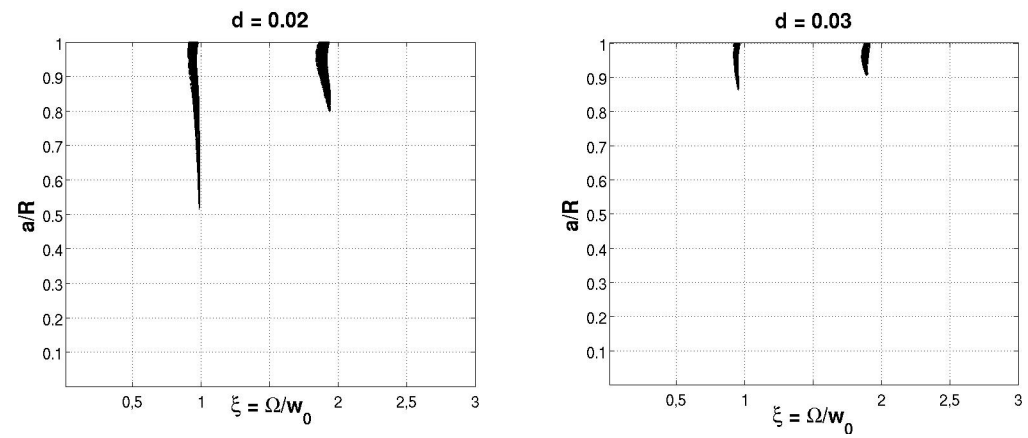

Fig. 13 Stable and unstable (hatched) zones evolution for $d=0.02$ and $d=0.03$

Mook [25], Nayfeh and Balachandran [24]). This method was used by Gasch [18], Meng and Gasch [23] and El Arem and Maitournam [14], El Arem and Nguyen [15] in the same context since it was developped to deal with linear stability of dynamical systems with periodic varying parameters. Also this method is easy to implement numerically.

Results for viscous dissipation $d \approx 2 \%$ and $d \approx 3 \%$, cf. Figure 13, show two principal instability areas: the first is located around the exact resonance $(\xi=1)$ and the second area (around $\xi=2$ ) corresponds to subharmonic resonance. It's important to note that even for weak viscous damping $(d \approx 2 \%)$ the stability of the cracked shaft is only slightly affected. When $d \approx 5 \%$ the zones of instabilities disappear completely for cracks with depth going to half the transverse section $\left(\frac{a}{R}=1\right)$. A third zone of instability has been observed by many authors at the subcritical speed range $(\xi<1.0)$ when considering a switching crack model (Gasch [18]) or in the case of very deep breathing cracks and low dissipation (El Arem and Nguyen [15]). But for more realistic operating conditions like in this article, as noticed by Patel and Darpe [28], El Arem [11], this zone is free of chaotic, quasi-periodic or subharmonic response. Since for real machines the viscous damping is $\approx 3 \%$, we can say that the effect of one propagating crack begins to threaten the stability only at important depth $\frac{a}{R}>0.85$ and this on a narrow interval around $\Omega \approx w_{0}$ and $\Omega \approx 2 \times w_{0}$. The passage through these frequencies must be done with the greatest care especially when it comes to turn off the machines after a long period of uninterrupted service.

\section{Conclusions}

In this article we have presented a systematic approach in dealing with the problem of modelling cracked rotating shafts. The breathing mechanism identification is the crucial step in the process and has to be made with the greatest care. We have spent years working on this very interesting subject and think that the approach presented here is original and that its implementation in industrial context is straightforward. The energy of the cracked element is written in a way to distinguish the additional part due to the crack presence. We concentrate all the nonlinearity of the problem 
in one single function whose identification thereafter is based on three-dimensional computations allowing a good representation of the crack behaviour. To make this approach generic, we have opted for the identification of a dimensionless flexibility so that it can be used in similar configuration. We have presented a good polynomial approximation of the flexibility for the case of cracks with rectilinear tip. This will avoid further three-dimensional computations for scientists or engineers who will have adopted this comprehensive approach.

Once the additional flexibility due to the crack identified, we have introduced it in a 2 dof dynamical system of a DeLaval rotor with a breathing crack at midspan. All the typical features related to cracked rotors have been observed. We have noticed the superharmonic resonnace phenomena when the machine is operated at an entier division of the first critical frequency. Also, we have found that the increase of the vibrational levels of the first and second superharmonics accompagned by the growth of static deflection are reliable indicators of a propagating crack.

We hope that the clarity of the different steps of the methodology presented here and the simplicity of its numerical implementation will make it the standard approach in dealing with cracked rotating shafts.

The next step will be to consider the case of multiple cracks affecting the same rotor at different positions along its axis. In this case, it would be wise to construct a beam-like finite element based on the development presented in this article. The new finite element will have a crack at midspan and will be inserted at the appropriate locations between classical beam elements to model a cracked region of the rotor. And this is what we are in the process of developing at this time by following the approach described by El Arem and Maitournam [14].

\section{References}

1. AL-Shudeifat MA, Butcher EA, Stern CR (2010) General harmonic balance solution of a cracked rotor-bearing-disk system for harmonic and sub-harmonic analysis: Analytical and experimental approach. International Journal of Engineering Science 48(10):921 - 935, structural Health Monitoring in the Light of Inverse Problems of Mechanics

2. Andrieux S, Varé C (2002) A 3d cracked beam model with unilateral contactapplication to rotors. European Journal of Mechanics, A/Solids 21:793-810

3. Bachschmid N, Pennacchi P, Tanz E (2007) Rotating shafts affected by transverse cracks: experimental behaviour and modelling techniques. Int $\mathrm{J}$ of Materials and Structural Integrity 1(1-2-3):71 - 116

4. Bachschmid N, Pennacchi P, Tanzi E (2008) Some remarks on breathing mechanism, on non-linear effects and on slant and helicoidal cracks. Mechanical Systems and Signal Processing 22(4):879 - 904

5. Bachschmid N, Tanzi E, Pennacchi P (2010) Cracked rotors: A survey on static and dynamic behaviour including modelling and diagnosis. Springer Berlin Heidelberg, DOI 10.1007/978-3-642-01485-7

6. Bachschmid N, Pennacchi P, Tanzi E (2011) Cracked Rotating Shafts: Typical Behaviors, Modeling and Diagnosis, Springer Netherlands, pp 441-454 
7. Chen C, Dai L (2007) Bifurcation and chaotic response of a cracked rotor system with viscoelastic supports. Nonlinear Dynamics 50(3):483-509

8. Darpe AK, Gupta K, , Chawla A (2004) Coupled bending, longitudinal and torsional vibrations of a cracked rotor. J Sound Vib 269:33-60

9. Dimarogonas AD (1996) Vibration of cracked structures: A state of the art review. Engineering Fracture Mechanics 55(5):831-857

10. Dimarogonas AD, Papadopoulos CA (1983) Vibration of cracked shafts in bending. Journal of Sound and Vibration 91(04):1583-593

11. El Arem S (2006) Vibrations non-lineaires des structures fissurées: application aux rotors de turbines (in french). PhD thesis, Ecole Nationale des Ponts et Chaussées

12. El Arem S (2009) Shearing effects on the breathing mechanism of a cracked beam section in bi-axial flexure. European Journal of Mechanics, A/Solids 28:1079-1087

13. El Arem S, Maitournam H (2007) Un élément fini de poutre fissurée: application à la dynamique des arbres tournants (in french). European Journal of Computational Mechanics 16(5):643-663

14. El Arem S, Maitournam H (2008) A cracked beam finite element for rotating shaft dynamics and stability analysis. Journal of Mechanics of Materials and Structures 3(5):893-910

15. El Arem S, Nguyen QS (2012) Nonlinear dynamics of a rotating shaft with a breathing crack. Annals of Solid and Structural Mechanics 3(1):1-14

16. El Arem S, Andrieux S, Varé C, Verrier P (2003) Loi de comportement en flexion d'une section de poutre fissurée avec prise en compte des effets de cisaillement (in french). In: 6ème colloque nationale en calcul des structures, Giens, vol II, pp 223-230

17. Floquet G (1879) Sur les équations différentielles linéaires coefficients périodiques. Ann Scien Ecole Nor Sup 2me série:3-132

18. Gasch R (1976) Dynamical behavior of a simple rotor with a cross-sectional crack. In: Vibrations in rotating machinery, I. Mech. E. Conference, London, pp 123-128

19. Gasch R (1993) A survey of the dynamic behavior of a simple rotating shaft with a transverse crack. J Sound and vibration 160:313-332

20. Grabowski B (1980) The vibrational behavior of a turbine rotor containing a transverse crack. Journal of Mechanical Design 102(1):140 - 146

21. Han Q, Chu F (2013) Parametric instability of a jeffcott rotor with rotationally asymmetric inertia and transverse crack. Nonlinear Dynamics 73(1):827-842

22. Mayes I, Davies W (1976) The vibrational behaviour of a rotating shaft system containing a transverse crack. In: Vibrations in rotating machinery, Inst. Mech. E. Conference, London, pp 53-65

23. Meng G, Gasch R (2000) Stability ans stability degree of a cracked flexible rotor supported on journal bearings. J Vibration and Acoustics, Transactions of ASME 122:116-125

24. Nayfeh AH, Balachandran B (1994) Applied nonlinear dynamics. Wiley series in nonlinear science

25. Nayfeh AH, Mook DT (1979) Nonlinear oscillations. John Wiley \& Sons 
26. Papadopoulos C (2004) Some comments on the calculation of the local flexibility of cracked shafts. Journal of Sound and Vibration 278:1205 - 1211

27. Papadopoulos CA (2008) The strain energy release approach for modeling cracks in rotors: A state of the art review. Mechanical Systems and Signal Processing 22(4):763 - 789

28. Patel TH, Darpe AK (2008) Influence of crack breathing model on nonlinear dynamics of a cracked rotor. J Sound and Vibration 311:953-972

29. Stoisser C, Audebert S (2008) A comprehensive theoretical, numerical and experimental approach for crack detection in power plant rotating machinery. Mechanical Systems and Signal Processing 22(4):818-844

30. Wauer J (1990) On the dynamics of cracked rotors: A literature survey. Applied Mechanical Reviews 43(1):13-17 